\title{
Investigation of Multi-Objective Optimization Criteria for RNA Design
}

\author{
David J. D. Hampson*, Sinem Sav ${ }^{\dagger}$, and Herbert H. Tsang \\ ${ }^{*}$ Applied Research Lab, Trinity Western University, Langley, British Columbia, Canada \\ ${ }^{\dagger}$ Faculty of Engineering \& Computer Science, Bilkent University, Ankara, Turkey \\ Email: herbert.tsang@twu.ca ${ }^{\ddagger}$
}

\begin{abstract}
RNA design is the inverse of RNA folding and it appears to be NP-hard. In RNA design, a secondary structure is given and the goal is to find a nucleotide sequence that will fold into this structure. To find such sequence(s) involves exploring the exponentially large sequence space. In literature, heuristic algorithms are the standard technique for tackling the RNA design. Heuristic algorithms enable effective and efficient exploration of the high-dimensional sequence-structure space when searching for candidates that fold into a given target structure. The main goal of this paper is to investigate the use of multi-objective criteria in SIMARD and Quality Pre-selection Strategy (QPS). The objectives that we optimize are Hamming distance (between designed structure and target structure) and thermodynamic free energy. We examine the different combinations of optimization criteria, and attempt to draw conclusions about the relationships between them. We find that energy is a poor primary objective but makes an excellent secondary objective. We also find that using multi-objective pre-selection produces viable solutions in far fewer steps than was previously possible with SIMARD.
\end{abstract}

\section{INTRODUCTION}

The RNA design problem is one of the NP-Hard problems [1] in the field of bioinformatics and it refers to the procedure of determining an RNA primary sequence given its secondary structure. Thus, it is the reverse of RNA secondary structure prediction. As the function of RNA is determined by its secondary structure, researchers in the field are interested in this problem to pave the way for new biotechnology and medicine researches such as customized drug design.

RNA secondary structure prediction is a well studied computational problem. In general, there are two types of approaches to study RNA secondary structures: 1) the single sequence approaches which predict the secondary structure based on experimentally determined energy parameters and 2) comparative sequence analysis approaches that try to improve their results by using functionally related sequences. The most popular single sequence approach to structure prediction methodology is the minimum free energy method. Among the different approaches, heuristic algorithms are a very popular and successful approach when compared to other deterministic approach. Our lab has developed a heuristic algorithm to predict RNA secondary structure and it has shown good prediction result even in pseudoknotted structures [2] [3] [4] [5].

Most of the RNA design and prediction problems are structured as optimization problems. In general, they require a long run-time. The obvious goal for improvement will be to decrease the run-time and to increase the quality of the solution at the same time.

Recently, we have introduced SIMARD, a RNA prediction algorithm based on simulated annealing [6]. SIMARD is structured as a single-objective optimization problem and it minimized over Hamming distance between the designed structure and desired structure. We have seen promising results by using Hamming distance only.

In this paper, we will utilize the simulated annealing framework in SIMARD, and additionally incorporate a multiobjective optimization approach. We will examine the result of employing both Hamming distance and free energy objectives. In the subsequent sections, the method, experimental setup, data used and results will be expanded on in detail.

\section{Multi-Objective Optimization}

\section{A. Computational Intelligence for RNA Prediction and Design}

Current algorithms in the field for RNA prediction and design are generally using global/local sampling methods, dynamic programming, stochastic searches, evolutionary algorithms and context free grammars. Most of them lack a robust optimization methodology as our previous experiments suggest [6]. Thus, they generally suffer from lack of reasonable run-time or better results.

Most RNA design algorithms are based on the heuristic approach. Some of the well-known RNA design packages are RNA-SSD [7] and INFO-RNA [8] using local stochastic searches, RNA-ensign [9] and improved version of RNAensign called IncaRNAtion [10] to decrease time complexity using global sampling methodology, MODENA [11], Frnakestein [12], GGI-FOLD [13], ERD [14] and our algorithm SIMARD [6] using evolutionary algorithms, incaRNAfbinv [15] using fragment-based design, and lastly antaRNA [16] which uses the ant colony optimization technique. Recently, some researchers has developed an online game to help with the RNA design problem [17]. They had summarized the design efforts of tens of thousands of human participants and three automated algorithms (RNAInverse, INFO-RNA and RNA-SSD) to generate some design principles.

\section{B. Using MOO for RNA Design}

Multi-objective optimization (MOO) is a paradigm which optimizes more than one objective at the same time [18]. 
When the number of objectives increase or there are conflicting objectives, optimizing them gets more difficult.

The rationale behind using MOO criteria for RNA design comes from our previous findings as a result of SIMARD experiments. We found that there are two separate objectives to optimize in RNA Design problem: Hamming distance and free energy. However, when we used simulated annealing (SA) technique for optimization, we had to choose one of them to optimize. Also, our experiments showed that when we choose Hamming distance as an objective, free energy increases as we ignore it in the optimization step. Similarly, when we use energy as an optimization objective, Hamming distance increases. These results brought using $\mathrm{MOO}$ as a new criteria for RNA Design to optimize both Hamming distance and free energy to our mind.

The objectives of this paper are as follows:

- To explain the methodology behind SIMARD with and without Quality Pre-selection Strategy (QPS).

- To give experiment setup and data together with the importance of two criteria: Hamming distance and free energy.

- To show the correlations

- To observe the impact of using Multi-Objective Optimization (MOO).

\section{METHOD}

The main RNA design framework for our algorithm is SIMARD (Simulated Annealing RNA Design) [6]. SIMARD uses an optimization strategy to find the optimal solution. In the following sections, we will describe SIMARD framework with Quality Pre-selection Strategy (QPS). This setup was described in detail in our other paper [19]. The contribution of this paper will be to show modification of SIMARD to accommodate multi-objective modification, including optimization based on thermodynamic energies and Hamming distance.

\section{A. SIMARD with $Q P S$}

SIMARD is a heuristic algorithm to design RNA secondary structure. It uses Simulated Annealing in order to find an optimal solution before the terminal condition is met. Algorithm 1 shows pseudo-code for SIMARD. After a starting solution is generated, a mutation is made to it and its fitness is evaluated based on given criteria. The solution is accepted or rejected based on its fitness and the current temperature of the algorithm. The temperature is reduced over time, moving the nature of the algorithm from exploration to exploitation.

Quality Pre-selection Strategy (QPS) is a mutation operator for SIMARD that generates a given number of sequences and returns the best based on given optimization criteria, discarding the dominated sequences. Algorithm 2 shows pseudocode for QPS. QPS is flexible in two main areas: pool size and optimization criteria. Pool size refers to the number of sequences considered per step. The more sequences we generate the more expensive the mutation operator is, but the quality of the best solution is generally higher due to a larger sample size. Optimization criteria refers to the standard by

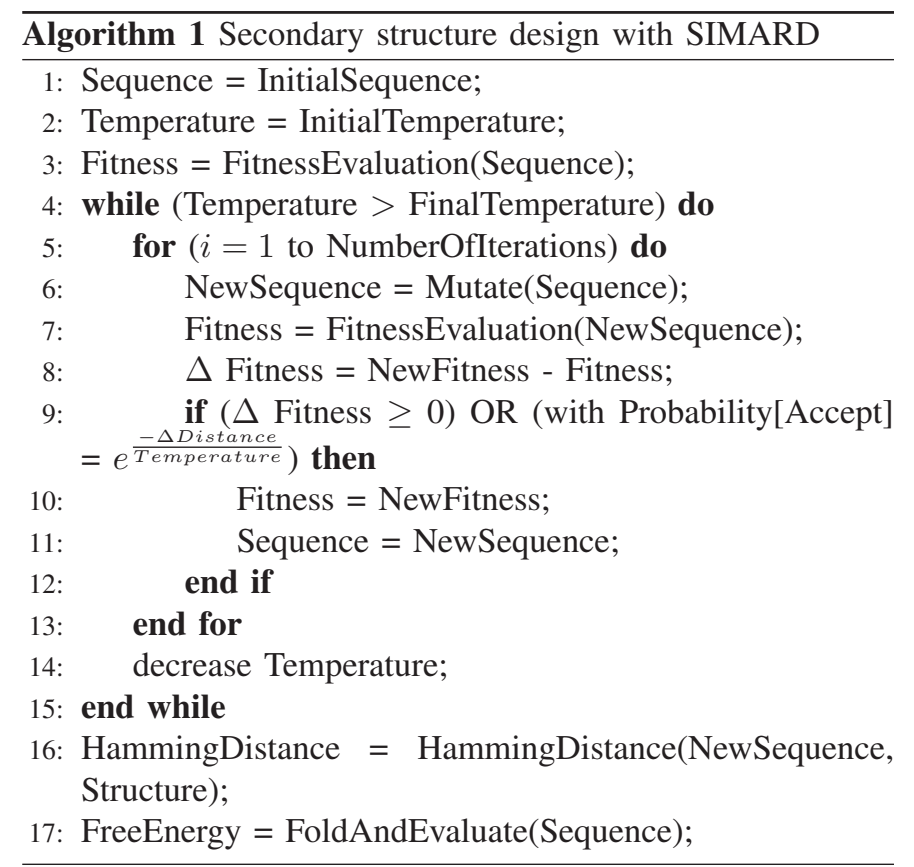

which each sequence is judged. The two main criteria that could be considered are Hamming distance to target structure and free energy.

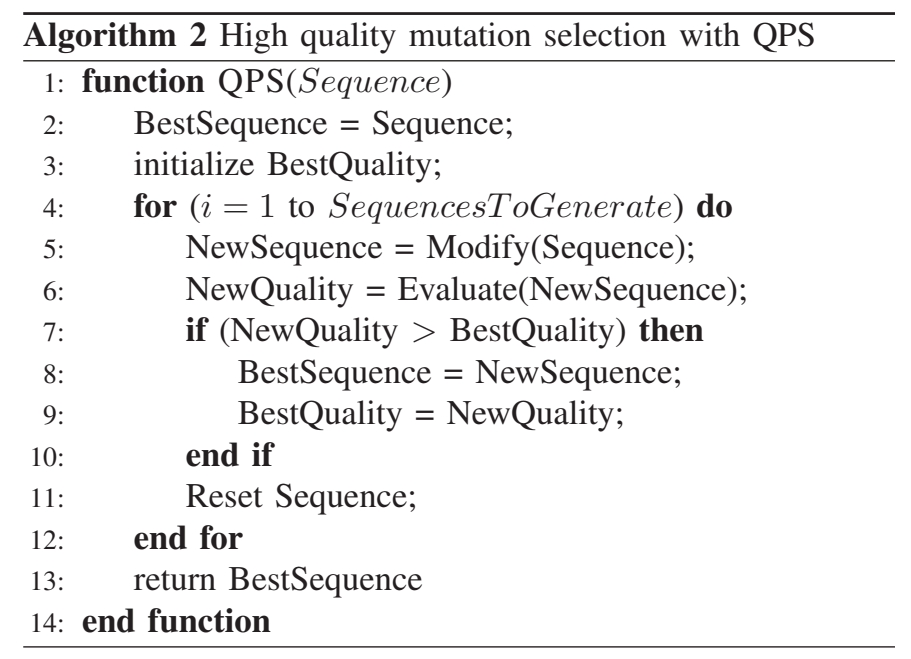

\section{B. Optimization Combinations}

When performing the optimization there are two criteria that we examine under the Multi-objective optimization (MOO) paradigm. These two criteria are: Hamming distance to target structure and free energy. There are a number of different combinations of optimization that we can test with our combination of pre-selection and SA acceptance.

1) Hamming distance: Hamming distance is a measure of the difference between two strings. Equation 1 specified the calculation of the hamming between two sequences $A_{i}$ and $B_{i}$, where $N$ is the total length of the strings.

$$
\text { Hamming_Distance }=\sum_{i=o}^{N-1}\left|A_{i}-B_{i}\right|
$$




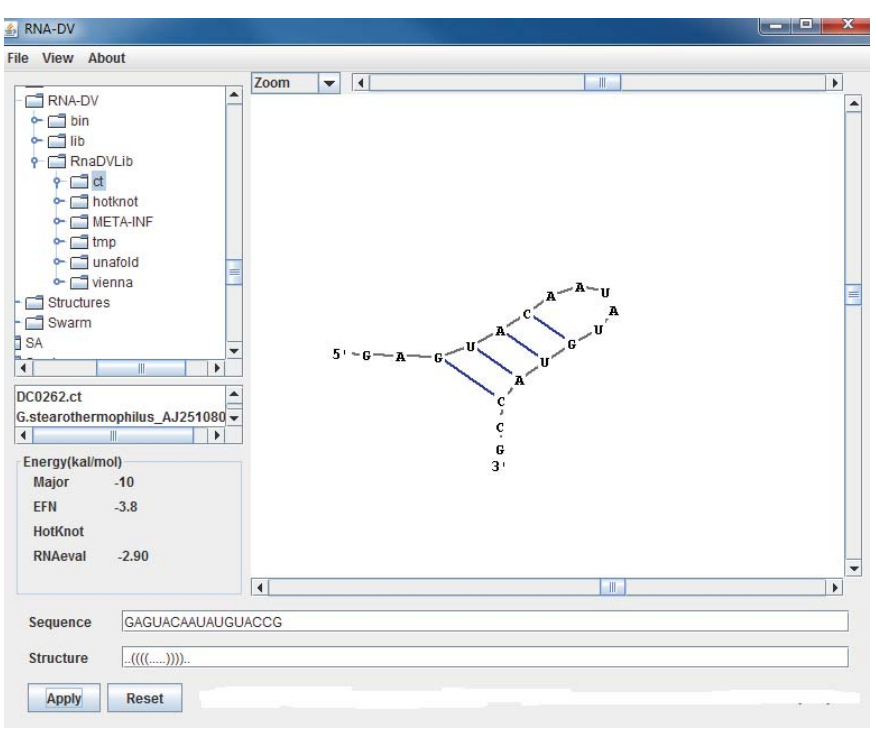

Fig. 1. RNA secondary structure expressed visually with dot bracket notation shown below. Image was generated using $R N A-D V$ software [20]

Figure 1 is showing the visualization of RNA secondary structure in both 2D structure and dot bracket format. The dot bracket format is a string composed of three symbols: dots, opening parentheses, and closing parentheses. This makes computing the Hamming distance of two structures very straight forward. Using this metrics, we can get a measure of how similar our designed structure is to the target structure.

In our case, we want to minimize Hamming distance to target structure, as a lower Hamming distance means that our working structure is closer to folding into the right shape. A Hamming distance to target structure of zero means that our working structure is identical to our target structure. This is one of our terminal conditions.

2) Free energy: Free energy is a measure of how stable a structure is. The lower the free energy, the more stable the structure. Most RNA secondary structure prediction and design algorithms are based on free energy minimization techniques, SIMARD can heuristically search for the structure with a free energy close to the minimum free energy $\Delta G$ for a strand of RNA, within given constraints.

SIMARD uses the thermodynamic energy model from Vienna RNA package [21]. For the purposes of this paper, the process of calculating the energy can be considered as a black box. The most important thing to realize is that we want a structure with as low free energy as possible. It is important to note that there are many ways any given RNA primary sequence can fold but we only consider the lowest possible free energy structure.

3) Correlation and problems: In our previous studies, we generally examined thermodynamic energy and Hamming distance separately as the optimization parameters [6] [19]. However, according to our trials, Hamming distance to target structure and free energy have a negative correlation. We calculated a correlation coefficient of -0.699 from 235,226 analyzed sequences produced by SIMARD. The sequences were all generated within the first 10,000 steps of the algorithm, where its behaviour is still fairly unbiased due to its exploratory nature. This negative correlation is easy to see when shown a plot of Hamming distance to target structure and free energy over the course of a SIMARD run. Figure 2 shows this. Without QPS, whichever objective is being optimized gradually decreases, while the other gradually increases.

In summary, because of this unexpected relationship between thermodynamic energy and Hamming distance, we propose to optimize based on both criteria. Table I shows the options of the different combination of the two criteria that we explored for this paper. While there are many other possibilities to explore, these are the ones we felt had the most promise, and would give us the best idea of the relationships between these values.

TABLE I

OPTIMIZATION COMBINATIONS BETWEEN THE TWO CRITERIA (HAMMING DISTANCE AND THERMODYNAMIC ENERGY).

\begin{tabular}{|l|l|l|}
\hline SIMARD optimization & QPS optimization & $\begin{array}{l}\text { QPS } \\
\text { pool } \\
\text { size }\end{array}$ \\
\hline Hamming distance & Energy & 2 \\
Energy & Hamming distance & 2 \\
Hamming distance & Multi-optimization method (Table II) & 2 \\
\hline Hamming distance & Energy & 3 \\
Energy & Hamming distance & 3 \\
Hamming distance & Multi-optimization method (Table II) & 3 \\
\hline
\end{tabular}

\section{Experiments Setup}

The primary goal of SIMARD is to produce a sequence with Hamming distance of zero to the target structure. However, without energy optimization from QPS, the system often returns a sequence with poor free energy. This is why our first experimental SIMARD configuration was to preselect an optimal energy sequence but accept it based on Hamming distance to target structure. Two variations of this were run: with QPS pool size 2, and with QPS pool size 3.

For our next experimental configuration, we preselected an optimal Hamming distance structure and accepted it based on energy. While we realize that it is not likely going to be a better solution, testing Hamming distance to target structure optimization in pre-selection (QPS) and SA optimization of energy can give us an idea of the relationship between the two objectives. We also ran two variations of this configuration: with QPS pool size 2, and with QPS pool size 3.

Finally, we present a novel multi-objective technique for pre-selection with QPS, described in Table II. In essence, we choose the best overall sequence - the one with the lowest relative Hamming distance to target structure and free energy. In a situation where sequence $\mathrm{A}$ has the lowest Hamming distance and the second lowest energy, and sequence B has the lowest energy and the second lowest Hamming distance, sequence A will be selected as Hamming distance is prioritized 


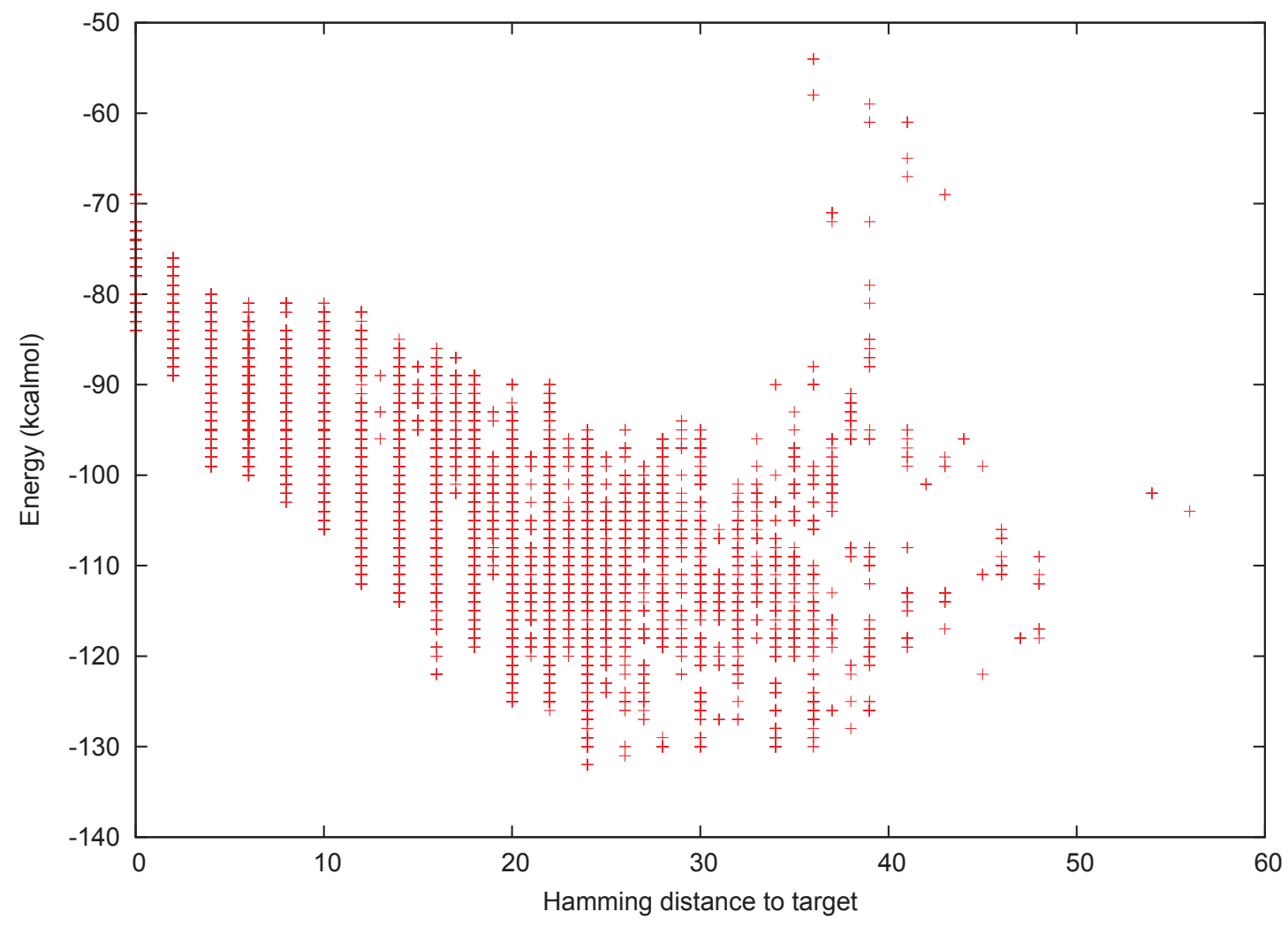

Fig. 2. Solutions generated in a run of SIMARD using energy as pre-selection criteria and Hamming distance to target as SA criteria. The horizontal axis represents Hamming distance and the vertical axis represents energy.

over energy. Once again, we also ran two variations of this configuration: with QPS pool size 2, and with QPS pool size 3.

TABLE II

MiNIMIZING MULTIPLE OBJECTIVES

\begin{tabular}{cccc}
\hline rank & hamming distance & free energy & ranking \\
\hline 1 & $\mathrm{~A}$ & $\mathrm{C}$ & $\mathrm{A}(1+2=3)$ \\
2 & $\mathrm{~B}$ & $\mathrm{~A}$ & $\mathrm{C}(3+1=4)$ \\
3 & $\mathrm{C}$ & $\mathrm{B}$ & $\mathrm{B}(2+3=5)$ \\
\hline
\end{tabular}

\section{Data}

We ran the experiments on sequences 1-30 from the Rfam dataset, excluding sequence RF00023 [22]. This is because RF00023 has lots of pseudo-knotted base pairs [12] The Rfam dataset contains sequences with lengths varying between $54-$ $451 \mathrm{nt}$ inclusive.

\section{RESUlTS AND Discussion}

Table III summarized the three sets of results.
TABLE III

THREE SETS OF EXPERIMENTS RESULTS.

\begin{tabular}{|l|l|l|}
\hline Result & Pre-selection & SA optimization \\
\hline 1 & Energy & Hamming distance \\
2 & Hamming distance & Energy \\
3 & Hamming distance & $\begin{array}{l}\text { Multi-objective strategy } \\
\text { outlined in Table II }\end{array}$ \\
\hline
\end{tabular}

A. Energy optimized in pre-selection, Hamming distance optimized in $S A$

In addition to having good Hamming distance, solutions produced by this technique show larege energy improvements over Vanilla SIMARD. A pool size of 3 sees a greater improvement of energy than a pool size of 2 .

However, as the algorithm runs, the higher the pool size, the more the energy increases near the end of the run (relative to where it was before). This is surprising behavior that may be caused by the strict pre-selection and exploratory SA optimization early on in the algorithm, forcing the search 


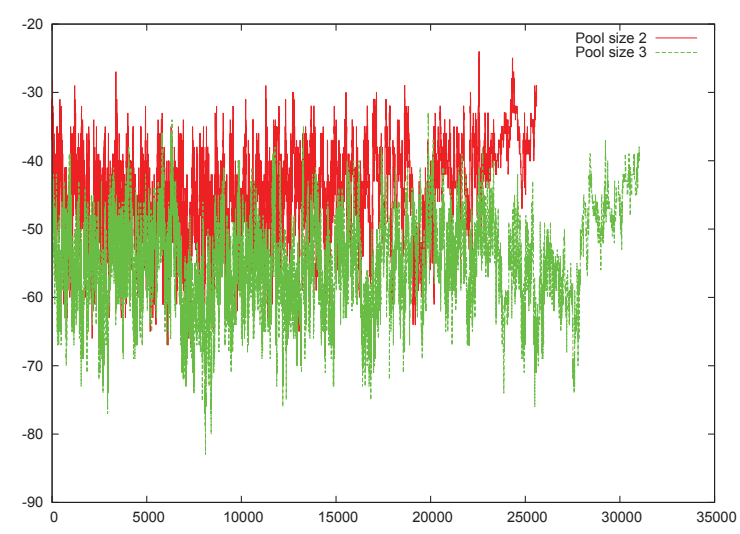

Fig. 3. Energy over two runs of the same sequence, RF00020, the 20th sequence in the RF dataset

space into a poor Hamming distance area, resolved by the exploitative nature of the late stage of SA optimization. If this is a case, a high pool size past a certain point is redundant as it just drives the algorithm from the optimal space at early stages, only to be overpowered by the strict SA optimization at late stages of the algorithm.

A segmented run may be the answer to this, as even though the run is driven back into a higher energy state, it is not driven all the way back up to its initial state. With this in mind, we could preselect energy for the early stages of the run, and then stop pre-selecting when the run got to a certain point.

It should be noted that the rise in energy is accompanied by a sharp fall in Hamming distance. This shows the negative correlation between the objectives in the case of this problem. Figure 3 shows the differences in early algorithm energy and the rise at the end of the algorithm.

Note that the sudden rise in energy is accompanied by a drop in Hamming distance. Also, the run with pool size 3 takes more steps. This is likely due to the negative correlation between Hamming distance to target structure and free energy, as the more greedy pre-selection tends to return sub-optimal Hamming distance solutions, making a longer road to one with Hamming distance of zero.

\section{B. Hamming distance optimized in pre-selection, energy opti- mized in $S A$}

While it is clear that this technique is not optimal for designing sequences, due to the larger final Hamming distance to target structure, it can be helpful in determining the connection between Hamming distance and energy. Our tests confirmed our hypothesis: that energy and Hamming distance don't have a very meaningful connection. There are a theoretically infinite amount of low energy structures, but they will not necessarily be the same structure as the target.

On the other hand, when paired with Hamming distance optimization, as is the case in the early phases of this algorithm (when SA is in an exploratory state and accepts almost every sequence), Hamming distance is optimized, the algorithm is guided towards the correct structure's low energy state. However, as the algorithm gets more and more greedy for low energy, it abandons the target structure and moves towards the closest free energy structure to its current state. Firure 5 shows this phenomenon around step 1,500. It is unlikely that an energy focused approach will ever be the optimal solution to the RNA design problem. Energy guided Hamming distance optimization, on the other hand, shows great promise. It is further explored in the following section.

\section{Multi Objective with pre-selection}

This technique found sequence with Hamming distance to target structure of zero surprisingly fast. Table V shows a comparison between the number of steps taken to terminate between the pool size 2 and 3 runs of this technique and the pool size 2 and 3 runs of the energy pre-selection technique(see Section IV-A). Fewer steps are required likely due to the fact that Hamming distance was optimized in pre-selection as well as in SA acceptance for this method, as opposed to only in SA acceptance for the other.

Table IV shows that free energy of solutions was also very low. A likely reason for energy being lower when the pool size was bigger could be that a bigger pool size skewed the overall optimization bias towards energy (since the pool of multiobjective optimization was growing but the SA Hamming optimization was not becoming more strict).

Because of how quickly this run terminates, we could try letting it run longer and increasing the weight of energy in the pre-selection phase. This would allow us to run it for however much time is available to us, continuing to optimize the solution further after a viable solution is found.

\section{CONCLUSION}

In this paper, we explored different SIMARD configurations using QPS. We looked at energy optimized in pre-selection and Hamming distance optimized with SA, as well as the opposite: Hamming distance optimized in pre-selection and energy optimized with SA.

From these two methods we learned that Hamming distance is a far better primary objective than energy as there are many low energy states that are not the same as a given target structure. We also confirmed that there is a negative correlation between those objectives in terms of this problem. Finally, we looked at a new multi-objective pre-selection technique, which proved to find a solution much faster than any other explored technique. It also outperformed vanilla SIMARD (no QPS) in terms of free energy.

As we have discussed in previous papers, a direction that we could go in would be to try a new secondary prediction structure (instead of Vienna Package). We can also look at trying to do a segmented run, or change the weight of the objectives in our multi-objective pre-selection. 


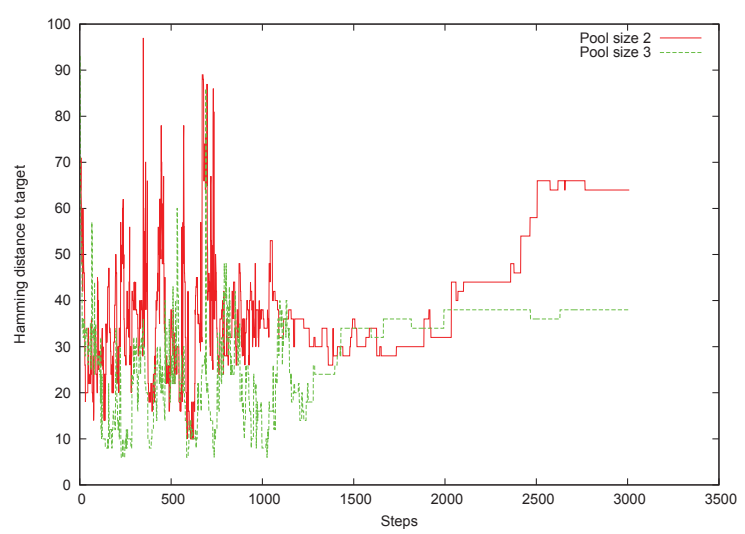

(a)

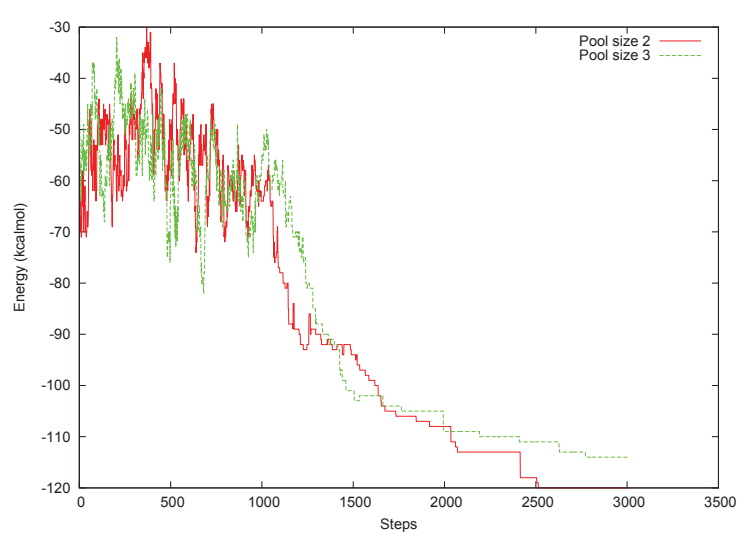

(b)

Fig. 4. SIMARD run with Hamming distance pre-selection and Energy SA optimization of sequence from RF dataset RF00025. The horizontal axis represents algorithmic steps and the vertical axis represents (a) Hamming distance to target structure (b) Free energy

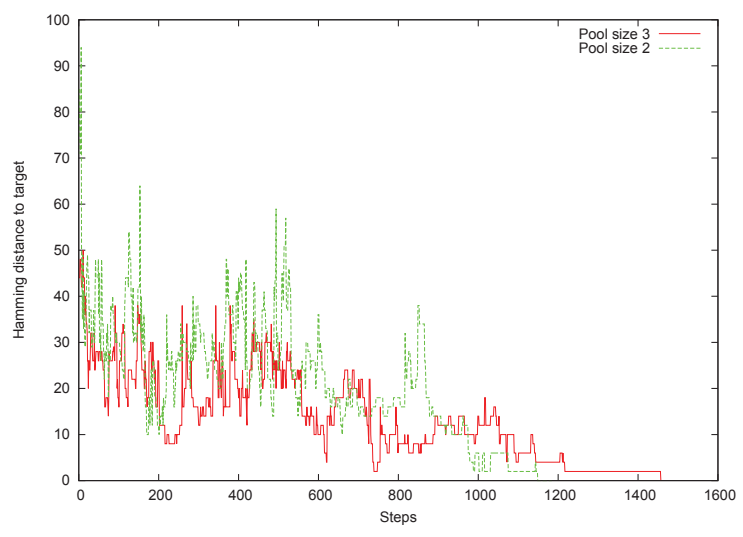

(a)

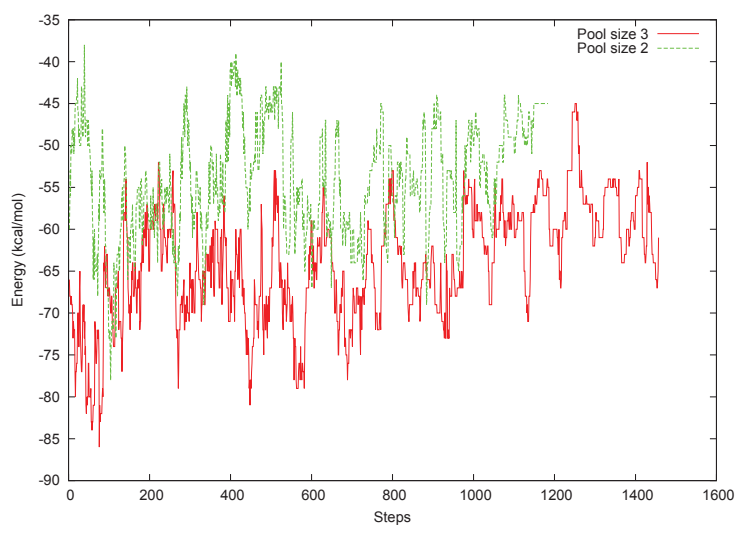

(b)

Fig. 5. SIMARD run with multi-objective pre-selection of sequence from RF dataset RF00025. The horizontal access represents algorithmic steps and the vertical axis represents (a) Hamming distance to target structure (b) Free energy

\section{ACKNOWLEDGMENT}

The authors would like to acknowledge support from Trinity Western University and Simon Fraser University. In addition, the authors would like to acknowledge the support from Mitacs Globalink project and a grant from the M.J. Murdock Charitable Trust Fund is also gratefully acknowledged. This research was enabled in part by support provided by WestGrid (www.westgrid.ca) and Compute Canada (www.computecanada.ca).

\section{REFERENCES}

[1] M. B. Schnall-Levin, "RNA : algorithms, evolution and design," Ph.D dissertation, Massachusetts Institute of Technology, 2011. [Online]. Available: http://hdl.handle.net/1721.1/67718
[2] H. H. Tsang and K. C. Wiese, "SARNA-Predict: Accuracy improvement of RNA secondary structure prediction using permutation based simulated annealing," IEEE/ACM Transactions on Computational Biology and Bioinformatics, vol. 99, no. 1, pp. 727-740, 2010.

[3] P. Grypma, J. Babbitt, and H. H. Tsang, "A study on the effect of different thermodynamic models for predicting pseudoknotted RNA secondary structures," in IEEE Symposium on Computational Intelligence in Bioinformatics and Computational Biology, 2013, pp. 52-59.

[4] P. Grypma and H. H. Tsang, "SARNA-Predict: Using adaptive annealing schedule and inversion mutation operator for RNA secondary structure prediction," in IEEE Symposium Series on Computational Intelligence, 2014, pp. 150-156.

[5] H. H. Tsang and K. C. Wiese, "A Permutation Based Simulated Annealing Algorithm to Predict Pseudoknotted RNA Secondary Structures," Int. J. Bioinformatics Res. Appl., vol. 11, no. 5, pp. 375-396, Sep. 2015. [Online]. Available: http://dx.doi.org/10.1504/IJBRA.2015.071938

[6] H. E. Erhan, S. Sav, S. Kalashnikov, and H. H. Tsang, "Examining the Annealing Schedules for RNA Design Algorithm," in Proceedings of the 
IEEE Congress on Evolutionary Computation, 2016, pp. 1295-1302.

[7] M. Andronescu, A. P. Fejes, F. Hutter, H. H. Hoos, and A. Condon, "A new algorithm for rna secondary structure design." Journal of Molecular Biology, vol. 336, no. 3, pp. 607-624, February 2004.

[8] A. Busch and R. Backofen, "INFO-RNAa server for fast inverse RNA folding satisfying sequence constraints," Nucleic Acids Research, vol. 35, pp. 310-313, 2007.

[9] A. Levin, M. Lis, Y. Ponty, C. W. O’Donnel, S. Devadas, B. Berger, and J. Waldispuhl, "A global sampling approach to designing and reengineering rna secondary structures," Nucleic Acids Research, vol. 40, 2012.

[10] V. Reinharz, Y. Ponty, and J. Waldispuhl, "A weighted sampling algorithm for the design of RNA sequences with targeted secondary structure and nucleotide distribution," ICMB/ECCB, vol. 29, pp. i308-i315, 2013.

[11] A. Taneda, "Multi-objective genetic algorithm for pseudoknotted RNA sequence design," Frontiers in Genetics, vol. 3, pp. 1-9, 2012.

[12] R. B. Lyngs, J. W. Anderson, E. Sizikova, A. Badugu, T. Hyland, and J. Hein, "Frnakenstein: multiple target inverse RNA folding," Frontiers in Genetics, vol. 3, pp. 1-12, 2012. [Online]. Available: http://www.biomedcentral.com/1471-2105/13/260

[13] M. Ganjtabesh, F. Zare-Mirakabad, and A. Nowzari-Dalini, "Inverse RNA Folding Solution Based on Multi-Objective Genetic Algorithm and Gibbs Sampling Method," EXCLI, vol. 2013, pp. 546-555, 2013.

[14] A. Esmaili-Taheri, M. Ganjtabesh, and M. Mohammad-Noori, "Evolutionary Solution for the RNA Design Problem," Bioinformatics, vol. 30 no. 9, pp. 1250-1258, 2014.

[15] M. Retwitzer, V. Reinharz, Y. Ponty, J. Waldispuhl, and D. Barash, "incaRNAfbinv: a web server for the fragment-based design of RNA sequences," Nucleic Acids Research, vol. 44, pp. 304-314, May 2016.

[16] K. Robert, M. Martin, and B. Rolf, "antaRNA - Ant Colony Based RNA Sequence Design,” Bioinformatics Advance Access, vol. 31, pp. 3114-3121, May 2015.

[17] J. Anderson-Lee, E. Fisker, V. Kosaraju, M. Wu, J. Kong, J. Lee, M. Lee, M. Zada, A. Treuille, and R. Das, "Principles for predicting $\{$ RNA $\}$ secondary structure design difficulty," Journal of Molecular Biology, vol. 428, no. 5, Part A, pp. 748 - 757, 2016.

[18] K. Deb, Multi-objective optimization using evolutionary algorithms. John Wiley \& Sons, 2001.

[19] S. Sav, D. J. Hampson, and H. H. Tsang, "SIMARD: A Simulated Annealing Based RNA Design Algorithm with Quality Pre-Selection Strategies," in IEEE Symposium Series on Computational Intelligence, 2016, p. submitted.

[20] H. H. Tsang and D. C. Dai, "RNA-DV: An interactive tool for editing and visualizing RNA secondary structures," in Proceedings of the ACM Conference on Bioinformatics, Computational Biology and Biomedicine, 2012, pp. 601-603.

[21] I. L. Hofacker, W. Fontana, F. S. Peter, L. S. Bonhoeffer, M. Tacker, and P. Schuster, "Fast Folding and Comparison of RNA Secondary Structures," Monatshefte fur Chemie, vol. 125, pp. 167-188, 1994. [Online]. Available: http://fontana.med.harvard.edu/www/Documents/WF/Papers/vienna.rna.pdf

[22] S. W. Burge, J. Daub, R. Eberhardt, J. Tate, L. Barquist, E. P. Nawrocki, S. R. Eddy, P. P. Gardner, and A. Bateman, "Rfam 11.0: 10 years of RNA families," Nucleic Acids Research, vol. 41, no. D1, pp. D226D232, 2013
TABLE IV

THE THERMODYNAMIC FREE ENERGY $\Delta$ G RESULT OF THE FINAL DESIGNED STRUCTURE. THE SEQUENCES ARE ARRANGED IN ORDER OF INCREASING LENGTH. BEST RESULTS ARE DENOTED IN BOLD.

\begin{tabular}{|c|c|c|c|c|}
\hline \multirow[b]{2}{*}{ Sequence name } & \multirow[b]{2}{*}{ Length } & \multicolumn{3}{|c|}{ Energy (kcal/mol) } \\
\hline & & $\begin{array}{l}\text { Multi objective } \\
\text { pool size } 2\end{array}$ & $\begin{array}{r}\text { pre-selection } \\
\text { pool size } 3\end{array}$ & Vanilla SIMARD \\
\hline RF0008 & 54 & -5 & -10 & -26 \\
\hline RF00029 & 73 & -11 & -16 & -8 \\
\hline RF0005 & 74 & -12 & -14 & -13 \\
\hline RF00027 & 79 & -24 & -29 & -25 \\
\hline RF00019 & 83 & -16 & -18 & -14 \\
\hline RF00014 & 87 & -23 & -27 & -15 \\
\hline RF0006 & 89 & -10 & -15 & -63 \\
\hline RF00026 & 102 & -2 & -2 & -1 \\
\hline RF0001 & 117 & -18 & -23 & -8 \\
\hline RF00021 & 118 & -27 & -38 & -20 \\
\hline RF00020 & 119 & -22 & -24 & -15 \\
\hline RF00016 & 129 & -13 & -15 & -31 \\
\hline RF00015 & 140 & -18 & -20 & -18 \\
\hline RF00022 & 148 & -21 & -30 & -18 \\
\hline RF0002 & 151 & -14 & -13 & -31 \\
\hline RF0007 & 154 & -31 & -36 & -23 \\
\hline RF0003 & 161 & -28 & -34 & -25 \\
\hline RF00013 & 185 & -31 & -47 & -15 \\
\hline RF0004 & 193 & -33 & -37 & -2 \\
\hline RF00025 & 210 & -22 & -30 & -23 \\
\hline RF00012 & 215 & -26 & -36 & -67 \\
\hline RF00017 & 301 & -70 & -99 & -65 \\
\hline RF00030 & 340 & -41 & -49 & -45 \\
\hline RF00028 & 344 & -32 & -41 & -29 \\
\hline RF0009 & 348 & -34 & -39 & -19 \\
\hline RF00010 & 357 & -65 & -79 & -14 \\
\hline RF00018 & 360 & -38 & -49 & -36 \\
\hline RF00011 & 382 & -68 & -75 & -28 \\
\hline RF00024 & 451 & -76 & -76 & -55 \\
\hline
\end{tabular}


TABLE V

THE NUMBER OF STEPS, OR ATTEMPTED SOLUTIONS, BEFORE THE TERMINARY CONDITION IS MET. RESULTS SHOWN ARE THE AVERAGE OF TWO RUNS PER SEQUENCE. THE SEQUENCES ARE ARRANGED IN ORDER OF INCREASING LENGTH. BEST RESULTS ARE DENOTED IN BOLD.

\begin{tabular}{rr|rrrr}
\hline \multirow{2}{*}{ Sequence name } & Length & \multicolumn{5}{|c}{ Algorithm } & \multicolumn{4}{c}{ Steps } \\
& & Multi objective & pre-selection & \multicolumn{2}{c}{ Energy pre-selection } \\
pool size 2 & pool size 3 & pool size 2 & pool size 3 \\
\hline RF0008 & 54 & $\mathbf{9 2}$ & $\mathbf{9 2}$ & 14419 & 30439 \\
RF00029 & 73 & $\mathbf{2 7 4}$ & 319 & 26033 & 19625 \\
RF0005 & 74 & $\mathbf{9 2}$ & $\mathbf{9 2}$ & 802 & 802 \\
RF00027 & 79 & $\mathbf{9 2}$ & $\mathbf{9 2}$ & 17222 & 10414 \\
RF00019 & 83 & $\mathbf{9 2}$ & 319 & 25279 & 19924 \\
RF00014 & 87 & $\mathbf{9 2}$ & 137 & 70509 & 71296 \\
RF0006 & 89 & $\mathbf{1 3 7}$ & 183 & 27235 & 26834 \\
RF00026 & 102 & 92 & $\mathbf{4 6}$ & 802 & 802 \\
RF0001 & 117 & $\mathbf{1 1 3 8}$ & 1366 & 28762 & 55865 \\
RF00021 & 118 & $\mathbf{9 2}$ & $\mathbf{9 2}$ & 30439 & 28837 \\
RF00020 & 119 & 2185 & $\mathbf{2 0 4 8}$ & 29534 & 23624 \\
RF00016 & 129 & 2139 & $\mathbf{2 0 4 8}$ & 8411 & 8411 \\
RF00015 & 140 & $\mathbf{3 6 5}$ & 547 & 53343 & 31640 \\
RF00022 & 148 & $\mathbf{1 8 3}$ & $\mathbf{1 8 3}$ & 1202 & 802 \\
RF0002 & 151 & $\mathbf{1 7 3 0}$ & 2231 & 33242 & 32441 \\
RF0007 & 154 & 729 & $\mathbf{6 3 8}$ & 19807 & 15739 \\
RF0003 & 161 & $\mathbf{2 2 3 0}$ & 2412 & 37247 & 35169 \\
RF00013 & 185 & $\mathbf{4 1 0}$ & 729 & 38878 & 32857 \\
RF0004 & 193 & $\mathbf{1 3 7}$ & 228 & 802 & 802 \\
RF00025 & 210 & $\mathbf{1 3 6 6}$ & 1411 & 13217 & 20026 \\
RF00012 & 215 & $\mathbf{3 1 9}$ & 410 & 36465 & 30350 \\
RF00017 & 301 & $\mathbf{1 3 2 0}$ & 1502 & 22429 & 18824 \\
RF00030 & 340 & $\mathbf{1 8 2 1}$ & 2003 & 48862 & 30439 \\
RF00028 & 344 & 2367 & $\mathbf{2 1 3 9}$ & 61678 & 51038 \\
RF0009 & 348 & 1821 & $\mathbf{1 6 3 9}$ & 8411 & 4406 \\
RF00010 & 357 & $\mathbf{2 6 4 1}$ & 3006 & 65311 & 65689 \\
RF00018 & 360 & $\mathbf{2 8 2 3}$ & 2868 & 25232 & 22028 \\
RF00011 & 382 & $\mathbf{2 5 4 9}$ & 2641 & 17222 & 15620 \\
RF00024 & 451 & $\mathbf{2 3 2 1}$ & 2958 & 36847 & 30439 \\
\hline & & & & &
\end{tabular}

